\title{
A Comment on the Article by L.V. Ksanfomality "Venus as a Natural Laboratory for Search of Life in High Temperature Conditions: Events on the Planet on March 1, 1982"
}

\author{
A. S. Spirin \\ Institute of Protein, Russian Academy of Sciences, Puschino, Moscow oblast, Russia \\ Department of Molecular Biology, Faculty of Biology, Moscow State University, Moscow, Russia \\ Received January 2, 2012
}

DOI: $10.1134 / \mathrm{S} 0038094612050103$

Being a molecular biologist, I certainly support the immediate publication of the article by L.V. Ksanfomality that is so "rebellious" from the standpoint of classical terrestrial biologists. Besides demonstrating the extremely unusual "strange stones" discovered on Venus, that, generally speaking, baffle the attempts of any purely "mineralogical" interpretation of the images obtained and their independent movement, the article seems to be very important in another respect. It begins the discussion about the principal physical possibility of the existence of large living, independently moving objects under very extreme temperature, pressure, and illumination conditions. The article estimates the actual physical parameters of conditions on the surface of Venus from the standpoint of the possibility that the functions immanent to such living organisms (independent movement, photosynthesis as an energy source, liquid water, that is, by the way, necessary for reproduction processes, if they are based on nucleic acids) are performed under these conditions. I have found nothing that would principally contradict the possibility that living organisms exist under Venusian conditions, including large ones that actively swim in its dense atmosphere and creep on its scorching surface.
In future, I would add at least two points for a serious scientific discussion. (1) Is it compulsory to suppose the necessity of photosynthesizing flora on Venus? And can Venus' "scorpions" or "horned owls" themselves possess the function of photosynthesis for the satisfaction of their energy needs, which are not so high? In addition, Venus is likely to have plenty of other energy sources, in particular, chemical sources (as, for example, in the situation in the deep ocean, completely deprived of light, with Earth microflora)? (2) Is it possible that nucleic acids exist and are structured under Venusian conditions, let us say, in some mineral water solutions, under corresponding temperatures and pressures? Then, this would solve the problem of reproduction, but no analogue of nucleic acids as an inheritance substance has been found anywhere as yet, or conceived.

\section{REFERENCES}

Ksanfomality, L.V., Venus as a Natural Laboratory for Search of Life in High Temperature Conditions: Events on the Planet on March 1, 1982, Solar Syst. Res., 2012, vol. 46 , no. 1 , pp. 41-53. 